\author{
ANNALES \\ POLONICI MATHEMATICI \\ XLII (1983)
}

\title{
Green's formula for right invertible operators
}

\author{
by D. Przeworska-Rolewioz (Warszawa)
}

\section{Dedicated to the memory of Jacek Szarski}

1. Preliminaries. Let $X$ be a linear space over a field $\mathscr{F}$ of scalars. In our further consideration we shall admit either $\mathscr{F}=\boldsymbol{R}$ or $\mathscr{F}=\boldsymbol{C}$. Let $L(X)$ be the set of all linear operators $A$ such that the domain of $A$ (denoted by $\operatorname{dom} A$ ) is a linear subset of $X$ and $A X \subset X$. In particular, we write: $L_{0}(X)=\{A \in L(X): \operatorname{dom} A=X\}$. Let $R(X)$ be the set of all right invertible operators belonging to $L(X)$. For a given $D \in R(X)$ we denote by $\mathscr{R}_{D}=\left\{R_{\gamma}\right\}_{\gamma \in \Gamma}$ the set of all right inverses of $D$. We shall assume that $\operatorname{dom} R_{\gamma}=X$ for $\gamma \in \Gamma$. Here and in the sequel we shall assume that $\operatorname{dim} \operatorname{ker} D>0$, i.e. $D$ is right invertible but not left invertible. Any element of $\operatorname{ker} D$ is a constant for $D$.

By $\mathscr{F}_{D}=\left\{F_{\gamma}\right\}_{\gamma \in \Gamma}$ we denote the set of all initial operators for $D$ (cf. [7]). By definition, $F$ is an initial operator for $D$ if is a projection onto $\operatorname{ker} D$ such that $F R=0$ for an $R \in \mathscr{R}_{D}$. This implies that $F$ is an initial operator if and only if there is an $R \in \mathscr{R}_{D}$ such that $F=I-R D$ on $\operatorname{dom} D$.

One can also prove (cf. [7]) that any projection $F$ onto $\operatorname{ker} D$ is an initial operator for $D$ corresponding to a right inverse $R=R_{0}-F R_{0}$ and that the definition of $R$ does not depend on the choice of the right inverse $R_{0}$.

We recall the Taylor-Gontcharov formula for right invertible operators. Let $N \in N$ and let $\left\{\gamma_{n}\right\} \subset \Gamma$ be arbitrarily fixed. Then the following identity holds on the domain of $D^{N}$ :

$$
I=F_{\gamma_{0}}+\sum_{k=1}^{N-1} R_{\gamma_{0}} \ldots R_{\gamma_{k-1}} F_{\gamma_{k}} D^{k}+R_{\gamma_{0}} \ldots R_{\gamma_{N-1}} D^{N}
$$

In particular, if $R_{\gamma_{k}}=R, F_{\gamma_{k}}=F$ for $k=0,1, \ldots, N-1$, then we obtain the Taylor formula:

$$
I=\sum_{k=0}^{N-1} R^{k} F D^{k}+R^{N} D^{N} \quad \text { on } \operatorname{dom} D^{N}
$$


For an arbitrary $x \in X$ the element $R_{\gamma} x(\gamma \in \Gamma)$ is a primitive element for $x$. One can prove that the difference of primitive elements is a constant (cf. [7]). Consider the operator: $F_{\beta} R_{\gamma}-F_{a} R_{\gamma}$ for arbitrarily fixed $\alpha, \beta$, $\gamma \in \Gamma$. This operator is independent of the choice of $R$ and plays the role of a definite integral for $D$. We can prove that

and that

$$
F_{\beta} R_{\gamma}-F_{a} R_{\gamma}=F_{\beta} R_{\alpha} \quad(\alpha, \beta, \gamma \in \Gamma)
$$

$$
F_{\beta} R_{\alpha} D=F_{\beta}-F_{\alpha} \quad(\alpha, \beta \in \Gamma)
$$

The last equality implies that a definite integral of an element is equal to the difference of initial values of a primitive element and that this constant is independent of the choice of a primitive element.

Let $A, B \in I(X)$ be arbitrary operators such that both superpositions $A B$ and $B A$ are well-defined. We shall write:

$$
[A, B]=A B-B A \text {. }
$$

Let $X$ be a linear ring (an algebra) over $\mathscr{F}$. We shall admit the following convention:

$$
[A, x]=A x-x A \quad \text { for all } x \in \operatorname{dom} A,
$$

i.e. $[A, x] y=(A x) y-x A y$ for all $x, y \in \operatorname{dom} A$.

Let $X$ be a commutative algebra (i.e. a commutative linear ring) over $\boldsymbol{R}$ and let $D \in R(X)$. $X$ is said be a $D$-algebra if the following condition is satisfied:

$$
x \in \operatorname{dom} D \text { and } y \in \operatorname{dom} D \quad \text { implies } \quad x y \in \operatorname{dom} D .
$$

Here and in the sequel we shall assume that $X$ is a $D$-algebra. Write:

$$
f_{D}(x, y)=D(x y)-c_{D}(x D y+y D x) \quad \text { for all } x, y \in \operatorname{dom} D,
$$

where:

(i) $c_{D}$ is a scalar dependent on $D$ only;

(ii) $f_{D}: \operatorname{dom} D \operatorname{dom} D \rightarrow \operatorname{dom} D$ is a bilinear and symmetric mapping dependent on $D$ only, i.e.

$$
f_{D}(y ; x)=f_{D}(x, y) \quad \text { for all } x, y \in \operatorname{dom} D \text {. }
$$

Using the denotation (1.2) we can write:

$$
D(x y)=c_{D}(x D y+y D x)+f_{D}(x, y) \quad \text { for } x, y \in \operatorname{dom} D .
$$

The bilinear operator $f_{D}$ will be called a non-Leibniz component. Non-Leibniz components for power of $D$ are determined by the following recursion formula:

$$
\begin{gathered}
f_{D}^{(0)}=0, \quad f_{D}^{(1)}=f_{D} \quad \text { and for } k=2,3, \ldots, x, y \in \operatorname{dom} D^{k} \\
f_{D}^{(k)}(x, y)=c_{D}^{k}\left[(D x)\left(D^{k-1} y\right)+\left(D^{k-1} x\right)(D y)\right]+ \\
+c_{D}^{k-1}\left[f_{D}^{(k-1)}\left(x, D^{k-1} y\right)+f_{D}^{(k-1)}\left(D^{k-1} x, y\right)\right]+D f_{D}^{(k-1)}(x, y) .
\end{gathered}
$$


The proof is given in [16]. Similar formulae hold for a superposition of right invertible operators. If $p \neq 0$ is an arbitrary real then $c_{p D}=c_{D}$, $f_{p D}=p f_{D}$.

Other properties of non-Leibniz components and several examples of $D$-algebras can be found also in [16].

2. Lagrange and Green formulae for polynomials in a right invertible operators with operator coefficients. Here and in the sequel we shall assume for $D$-algebras under consideration the denotation used in formula (1.7). We also always assume that $c_{D} \neq 0$ (cf. Example 2.8 in [16]).

THEOREM 2.1. Let $X$ be a D-algebra. Write

$$
Q(D)=\sum_{k=0}^{N} Q_{k} D^{k} ; \quad Q^{+}(D)=\sum_{k=0}^{N}(-1)^{k} D^{k} Q_{k}
$$

where $Q_{0}, Q_{1}, \ldots, Q_{N} \in L_{0}\left(\operatorname{dom} D^{N}\right), N \geqslant 1$ and $Q_{N}$ is an invertible operator (or identity). Then for every $x, y \in \operatorname{dom} D^{N}$ the following identity holds:

$$
\begin{aligned}
x Q(D) y-y Q^{+}(D) x= & \sum_{k=0}^{N}\left\{(-1)^{k+1} c_{D}^{-k}\left[D^{k}\left(y Q_{k} x\right)-f_{D}^{(k)}\left(Q_{k} x, y\right)\right]+\right. \\
& \left.+\left[x Q_{k}-\left(Q_{k} x\right)\right] D^{k} y+\left[1+(-1)^{k}\right]\left(Q_{k} x\right) D^{k} y\right\}
\end{aligned}
$$

Proof. Our assumptions imply that

$$
\begin{gathered}
x Q(D) y-y Q^{+}(D) x=\sum_{k=0}^{N}\left[x Q_{k} D^{k} y-y(-1)^{k} D^{k} Q_{k} x\right] \\
=\sum_{k=0}^{N}\left\{(-1)^{k+1}\left[\left(Q_{k} x\right) D^{k} y+y D^{k}\left(Q_{k} x\right)\right]+\left[x Q_{k} D^{k} y+(-1)^{k}\left(Q_{k} x\right) D^{k} y\right]\right\} \\
=\sum_{k=0}^{N}\left\{(-1)^{k+1} c_{D}^{-k}\left[D^{k}\left(y Q_{k} x\right)-f_{D}^{(k)}\left(Q_{k} x, y\right)\right]+\left[x Q_{k}-\left(Q_{k} x\right)\right] D^{k} y+\right. \\
\left.+\left[1+(-1)^{k}\right]\left(Q_{k} x\right) D^{k} y\right\}
\end{gathered}
$$

Formula (2.2) is the Lagrange formula for polynomials in $D$ with operator coefficients.

COROLLARY 2.1. Let $X$ be a D-algebra and let $Q(D)$ and $Q^{+}(D)$ be defined by formula (2.1). Then for all $F_{a}, F_{\beta} \in \mathscr{F}_{D}, R_{\alpha} \in \mathscr{R}_{D}(\alpha \neq \beta)$ and for all $x, y \in \operatorname{dom} D^{N}$ the following identity holds:

$$
\begin{aligned}
& F_{\beta} R_{a}\left[x Q(D) y-y Q^{+}(D) x\right] \\
& \qquad \sum_{j=0}^{N-1}(-1)^{j} e_{D}^{-(j+1)}\left(F_{-}-F_{a}\right) D^{j}\left(y Q_{j+1} x\right)+ \\
& \quad+F_{\beta} R_{a}\left[g_{Q(D)}(x, y)+h_{Q(D)}(x, y)\right],
\end{aligned}
$$


where

$$
\begin{aligned}
g_{Q(D)}(x, y)=\sum_{k=1}^{N}\left\{(-1)^{k} c_{D}^{-k} f_{D}^{(k)}\left(Q_{k} x, y\right)+\right. & \\
& \left.+\left[1+(-1)^{k}\right]\left(Q_{k} x\right) D^{k} y\right\}+y Q_{0} x
\end{aligned}
$$

$$
h_{Q(D)}(x, y)=\sum_{k=0}^{N}\left[x Q_{k}-\left(Q_{k} x\right)\right] D^{k} y
$$

Proof. Formulae (1.4), (2.2), (2.4), (2.5) together imply that for all $F_{a}, F_{\beta} \in \mathscr{F}_{D}, R_{\alpha} \in \mathscr{R}_{D}, \alpha \neq \beta$ and $x, y \in \operatorname{dom} D^{N}$ we have

$$
\begin{aligned}
& F_{\beta} R_{\mathrm{a}}\left[x Q(D) y-y Q^{+}(D) x\right] \\
& =F_{\beta} R_{a}\left\{-y Q_{0} x+\sum_{k=1}^{N}(-1)^{k+1} c_{D}^{-k}\left[D^{k}\left(y Q_{k} x\right)\right]+\sum_{k=0}^{N}(-1)^{k+2} c_{D}^{-k} f_{D}^{(k)}(x, y)+\right. \\
& \left.+\sum_{k=0}^{N}\left[x Q_{k}-\left(Q_{k} x\right)\right] D^{k} y+2\left(Q_{0} x\right) y+\sum_{k=1}^{N}\left[1+(-1)^{k}\right]\left(Q_{k} x\right) D^{k} y\right\} \\
& =F_{\beta} R_{\alpha}\left\{\sum_{j=0}^{N-1}(-1)^{j+2} c_{D}^{-(j+1)}\left[D^{j+1}\left(y Q_{j+1} x\right)\right] \sum_{k=0}^{N} c_{D}^{-k} f_{D}^{(k)}(x, y)+\right. \\
& \left.+\sum_{k=0}^{N}\left[x Q_{k}-\left(Q_{k} x\right)\right] D^{k} y+\sum_{k=1}^{N}\left[1+(-1)^{k}\right]\left(Q_{k} x\right) D^{k} y+y Q_{0} x\right\} \\
& =\sum_{j=0}^{N-1}(-1)^{j} c_{D}^{-(j+1)} F_{\beta} R_{a} D\left[D^{j}\left(y Q_{j+1} x\right)\right] F_{\beta} R_{\alpha}\left[g_{Q(D)}(x, y)+h_{Q(D)}(x, y)\right] \\
& =\sum_{\beta=0}^{N-1}(-1)^{j} c_{D}^{-(j+1)}\left(F_{\beta}-F_{\alpha}\right) D^{j}\left(y Q_{j+1} x\right)+F_{\beta} R_{a}\left[g_{Q(D)}(x, y)+h_{Q(D)}(x, y)\right]
\end{aligned}
$$

since $f_{D}^{(0)}=0$.

Formula (2.3) is the Green formula for polynomials in $D$ with operator coefficients. Now we shall specify coefficients. Observe that bilinear operators appearing in this formula map the space of constants into itself.

CoRoLlary 2.2. Suppose that all assumptions of Corollary 2.1 are satisfied. If $Q_{0}, \ldots, Q_{N}$ are operators of multiplication by elements of $X$, i.e.

$$
Q_{k} x=a_{k} x, \quad \text { where } a_{k} \in X(k=0,1, \ldots, N) \text { for } x \in X,
$$

then

$$
\begin{aligned}
Q(D) x=\sum_{k=0}^{N} a_{k} D^{k} x, \quad Q^{+}(D) x=\sum_{k=0}^{N}(-1)^{k} D^{k}\left(a_{k} x\right) \\
\text { for } x \in X, \quad h_{Q(D)}=0
\end{aligned}
$$


where $h_{Q(D)}$ is defined by (2.5), and the Green formula is of the form

$$
\begin{aligned}
F_{\beta} R_{a}[x Q(D) & \left.y-y Q^{+}(D) x\right] \\
= & \sum_{j=0}^{N-1}(-1)^{j} c_{D}^{-(j+1)}\left(F_{\beta}-F_{a}\right) D^{j}(a x y)+F_{\beta} R_{a} g_{Q(D)}(x, y)
\end{aligned}
$$

where

$$
g_{Q(D)}=\sum_{k=1}^{N}\left\{(-1)^{k} c_{D}^{-k} f_{D}^{(k)}\left(a_{k} x, y\right)+\left[1+(-1)^{k}\right] a_{k} x D^{k} y\right\}+a_{0} x y
$$

Indeed, by our definition for all $x, y \in \operatorname{dom} D^{N}$

$$
h_{Q(D)}(x, y)=\sum_{k=0}^{N}\left[x Q_{k}-\left(Q_{k} x\right)\right] D^{k} y=\sum_{k=0}^{N}\left[x a_{k}-a_{k} x\right] D^{k} y=0
$$

and $y Q_{0} x=a_{0} x y$.

CoRoLlaRY 2.3. Suppose that all assumptions of Corollary 2.1 are satisfied. If the coefficients $Q_{0}, \ldots, Q_{N}$ commute with $D: D Q_{k}=Q_{k} D$ $(k=0,1, \ldots, N)$, then

$$
Q^{+}(D)=Q(-D)=Q\left(D^{+}\right), \quad \text { where } D^{+}=-D .
$$

Indeed, by our assumptions we have

$$
Q^{+}(D)=\sum_{k=0}^{N}(-1)^{k} D^{k} Q_{k}=\sum_{k=0}^{N} Q_{k}(-D)^{k}=Q(-D)=Q\left(D^{+}\right)
$$

COROLLARY 2.4. Suppose that all assumptions of Corollary 2.1 are satisfied. If the coefficients of $Q(D)$ are scalars, i.e. if $Q_{k}=q_{k} I$, where $q_{k} \in \boldsymbol{R}$ $(k=0,1, \therefore, N), q_{N} \neq 0$, then

$$
Q^{+}(D)=Q(-D)=Q\left(D^{+}\right)
$$

for all $x, y \in \operatorname{dom} D^{N}$

$$
\begin{aligned}
& g_{Q(D)}(x, y)=\sum_{k=1}^{N} q_{k}\left\{(-1)^{k} c_{D}^{-k} f_{D}^{(k)}(x, y)+\left[1+(-1)^{k}\right] x D^{k} y\right\}+q_{0} x y, \\
& h_{Q(D)}(x, y)=0
\end{aligned}
$$

and the Green formula is of the form

$$
\begin{aligned}
F_{\beta} R_{\alpha}[x Q(D) y-y Q(-D) x] \\
=\sum_{j=0}^{N-1}(-1)^{j} c_{D}^{-(j+1)} g_{j}\left(F_{\beta}-F_{a}\right) D^{j}(x y)+F_{\beta} R_{a} g_{Q(D)}(x, y) .
\end{aligned}
$$


Indeed, since $Q_{0}, \ldots, Q_{N}$ commute with $D$, Corollary 2.3 implies that $Q^{+}(D)=Q(-D)$. Moreover, we have

$$
\begin{aligned}
g_{Q(D)}(x, y) & =\sum_{k=1}^{N}\left\{(-1)^{k} c_{D}^{-k} f_{D}^{(k)}\left(q_{k} x, y\right)+\left[1+(-1)^{k}\right] q_{k} x D^{k} y\right\}+q_{0} x y \\
& =\sum_{k=1}^{N} q_{k}\left\{(-1)^{k} c_{D}^{-k} f_{D}^{(k)}(x, y)+\left[1+(-1)^{k}\right] x D^{k} y+q_{0} x y\right\}
\end{aligned}
$$

since $f_{D}^{(k)}$ are bilinear operators. We also find

$$
h_{Q(D)}(x, y)=\sum_{k=0}^{N}\left[x Q_{k}-Q_{k} x\right] D^{k} y=\sum_{k=0}^{N} q_{k}(x-x) D^{k} y=0
$$

Therefore the Green formula in our case is of the form (2.8).

CoRollaRY 2.5. Suppose that all assumptions of Corollary 2.4 are satisfied. Then the Green formula (2.9) can be written as follows:

$$
\begin{aligned}
& F_{\beta} R_{a}[x Q(D) y-y Q(-D) x] \\
= & c_{D}^{-1}\left(F_{\beta}-F_{a}\right)\left[Q\left(-o_{D}^{-1} D\right)-(-1)^{N} q_{N} D^{N}\right](x y)+F_{\beta} R_{a} g_{Q(D)}(x, y)
\end{aligned}
$$

for all $x, y \in \operatorname{dom} D^{N}$, where $g_{Q(D)}$ is as in Corollary 2.4 .

Indeed, formula (2.8) implies that for all $x, y \in \operatorname{dom} D^{N}$

$$
\begin{aligned}
& F_{\beta} R_{a}[x Q(D) y-y Q(-D) x] \\
= & \sum_{j=0}^{N-1}(-1)^{j} c_{D}^{-(j+1)} q_{j}\left(F_{\beta}-F_{a}\right) D^{j}(x y)+F_{\beta} R_{a} g_{Q(D)}(x, y) \\
= & c_{D}^{-1}\left(F_{\beta}-F_{a}\right) \sum_{j=0}^{N-1} q_{j}(-1)^{j} c_{D}^{-j} D^{j}(x y)+F_{\beta} R_{a} g_{Q(D)}(x, y) \\
= & c_{D}^{-1}\left(F_{\beta}-F_{a}\right)\left[Q\left(-c_{D}^{-1} D\right)(x y)-(-1)^{N} q_{N} D^{N}\right](x y)+F_{\beta} R_{a} g_{Q(D)}(x, y) .
\end{aligned}
$$

If $c_{D}=1$, then formula (2.9) is of the form

$$
\begin{aligned}
F_{\beta} R_{\alpha} & {[x Q(D) y-y Q(-D) x] } \\
& =\left(F_{\beta}-F_{\alpha}\right) Q(-D)-(-1)^{N} q_{N} D^{N}(x y)+F_{\beta} R_{a} g_{Q(D)}(x, y),
\end{aligned}
$$

where

$$
g_{Q(D)}(x, y)=\sum_{k=1}^{N} q_{k}\left\{(-1)^{k} f_{D}^{(k)}(x, y)+\left[1+(-1)^{k}\right] x D^{k} y\right\}+q_{0} x y
$$

CoRollary 2.6. Suppose that all assumptions of Corollary 2.1 are satisfied. Let $x, y$ be solutions of equations

$$
Q(D) y_{-}=v, \quad Q^{+}(D) x=u,
$$


respectively, where $u, v \in X$ are given. Then

(2.13) $\quad F_{\beta} R_{a}(x v-y u)$

$$
=\sum_{j=0}^{N-1}(-1)^{j} c_{D}^{-(j+1)}\left(F_{\beta}-F_{a}\right) D^{j}\left(y Q_{j+1} x\right)+F_{\beta} R_{a}\left[g_{Q(D)}(x, y)+h_{Q(D)}(x, y)\right] \text {. }
$$

The proof immediately follows from formula (2.3).

CoROLLARY 2.7. Let $X$ be a $D$-algebra. Let $N$ be an arbitrarily fixed positive integer and let $x, y \in \operatorname{dom} D^{N}$. Then the Lagrange formula for the operator $D^{N}$ is of the form

$$
\begin{aligned}
x D^{N} y-y\left(D^{+}\right)^{N} x= & (-1)^{N+1} c_{D}^{-N} D^{N}(x y)-f_{D}^{(N)}(x, y)+ \\
& +\left[1+(-1)^{N}\right] x D^{N} y, \quad \text { where } D^{+}=-D
\end{aligned}
$$

and the Green formula is of the form

$$
\begin{array}{r}
F_{\beta} R_{a}\left[x D^{N} y-y\left(D^{+}\right)^{N} x\right]=(-1)^{N+1} c_{D}^{-N}\left(F_{\beta}-F_{a}\right) D^{N-1}(x y)+ \\
+(-1)^{N} c_{D}^{-N} f_{D}^{(N)}(x, y)+\left[1+(-1)^{N}\right] x D^{N} y
\end{array}
$$

where $F_{a}, F_{\beta} \in \mathscr{F}_{D}, R_{a} \in \mathscr{R}_{D}, \beta \neq \alpha$.

Indeed, if we put in Theorem 2.1 and Corollary $2.1 Q_{0}=Q_{1}=\ldots$ $\ldots=Q_{N-1}=0, Q_{N}=I$ then we obtain

$$
Q(D)=D^{N}, \quad Q^{+}(D)=(-1)^{N} D^{N}=\left(D^{+}\right)^{N} .
$$

CoRollary 2.8. Suppose that $D_{1}, D_{2} \in R(X), D=D_{1} D_{2}$ and $X$ is a D-algebra. Then the Lagrange and Green formulae for the superposition $D=D_{2} D_{1}$ are of the form: for all $x, y \in \operatorname{dom} D$

$$
\begin{aligned}
& x D_{2} D_{1} y-y D_{2}^{+} D_{1}^{+} \\
& =c_{D_{1}}^{-1} c_{D_{2}}^{-1}\left\{D_{2} D_{1}(x y)-f_{D_{2}}(x, y)-D_{2} f_{D_{1}}(x, y)+\right. \\
& \left.+c_{D_{1}} c_{D_{2}}\left[\left(D_{1} x\right)\left(D_{2} y\right)+\left(D_{2} x\right)\left(D_{1} y\right)\right]\right\} \\
& F_{\beta} R_{a}\left(x D_{2} D_{1} y-y D_{2}^{+} D_{1}^{+}\right) \\
& =c_{D_{1} D_{2}}\left(F_{\beta}-F_{\alpha}\right)(x y)-c_{D_{1} D_{2}} F_{\beta} R_{a}\left\{f_{D_{2}}(x, y)+D_{2} f_{D_{1}}(x, y)+\right. \\
& \left.+c_{D_{1}} c_{D_{2}}\left[\left(D_{1} x\right)\left(D_{2} y\right)+\left(D_{2} x\right)\left(D_{1} y\right)\right]\right\} \text {, }
\end{aligned}
$$

where we admit $D_{1}^{+}=-D_{1}, D_{2}^{+}=-D_{2}, D^{+}=-D$ and

$$
\begin{aligned}
F_{\beta} R_{\alpha}= & {\left[F_{\beta}^{(1)} R_{\alpha}^{(1)}+R_{\beta}^{(1)} F^{(2)}\right] R_{a}^{(2)}, } \\
& F_{a}^{(i)}, F_{\beta}^{(i)} \in \mathscr{F}_{D_{i}} ; R_{a}^{(i)}, R_{\beta}^{(i)} \in \mathscr{R}_{D_{i}}(i=1,2) \text { and } \beta \neq \alpha .
\end{aligned}
$$

Indeed, $D^{+}=-D_{2}^{+} D_{1}^{+}=-\left(-D_{2}\right)\left(-D_{1}\right)=-D_{2} D_{1}=-D$.

It is well known [8] that

$$
\begin{gathered}
F_{\beta}=F_{\beta}^{(1)}+R_{\beta \beta}^{(1)} F^{(2)} D_{1} \in \mathscr{F}_{D}, \quad F_{\alpha}=F_{\alpha}^{(1)}+R_{a}^{(1)} F_{\alpha}^{(2)} D_{1} \in \mathscr{F}_{D}, \\
R_{\alpha}=R_{a}^{(1)} R_{\alpha}^{(2)} \in \mathscr{R}_{D}
\end{gathered}
$$

19 - Annales Polonicl Mathematici XuII 
Hence

$$
\begin{gathered}
F_{\beta} R_{\alpha}=\left(F_{\beta}^{(1)}+R_{\beta}^{(1)} F_{\beta}^{(2)} D_{1}\right) R_{a}^{(1)} R_{\alpha}^{(2)}=\left[F_{\beta}^{(1)} R_{\alpha}^{(1)}+R_{\beta}^{(1)} F_{\beta}^{(2)}\right] R_{a}^{(2)} \\
F_{\beta}-F_{\alpha}=F_{\beta}^{(1)}-F_{a}^{(1)}+\left(R_{\beta}^{(1)} F_{\beta}^{(2)}-R_{a}^{(2)} F_{a}^{(2)}\right) D_{1} .
\end{gathered}
$$
$\epsilon \operatorname{dom} D$

Since $D^{+}=-D$, formulae (2.2) and (2.3) imply that for all $x, y$

$$
\begin{gathered}
x D y-y D^{+} x=x D y+y D x=c_{D}^{-1}\left[D(x y)-f_{D}(x, y)\right], \\
F_{\beta} R_{a}\left(x D y-y D^{+} x\right)=F_{\beta} R_{a}\left\{c_{D}^{-1} D(x y)-f_{D}(x, y)\right\} \\
=c_{D}^{-1} F_{\beta} R_{a} D(x y)-c_{D}^{-1} F_{\beta} R_{a} f_{D}(x, y) \\
=c_{D}^{-1}\left(F_{\beta}-F_{a}\right)(x y)-c_{D}^{-1} F_{\beta} R_{\alpha} f_{D}(x, y) .
\end{gathered}
$$

This implies the required formulae (2.16) and (2.17).

Corollary 2.9. Suppose that $D_{1}, D_{2} \in R(X), D=D_{2}^{q} D_{1}^{p}, p, q \in N$, are arbitrarily fixed and $X$ is a D-algebra. Then we have

$$
c_{D}=c_{D_{1}}^{p} c_{D_{2}}^{q}
$$

$$
f_{D}(x, y)=f_{D_{2}}^{(q)}(x, y)+D_{2}^{q} f_{D_{1}}^{(p)}(x, y)+
$$$$
+c_{D_{1}}^{p} c_{D_{2}}^{q}\left[\left(D_{1}^{p} x\right)\left(D_{2}^{q} y\right)+\left(D_{2}^{q} x\right)\left(D_{1}^{p} y\right)\right] \quad \text { for } x, y \in \operatorname{dom} D
$$

(2.21) $D^{+}=(-1)^{p+q} D$,

$$
F_{\gamma}=\sum_{k=0}^{p-1} R_{\gamma}^{(1) \ddot{k}} F^{(1)} D_{1}^{R}+\left(R_{\gamma}^{(1)}\right)^{p} \sum_{j=0}^{q-1}\left(\dot{R}_{\gamma}^{(2)}\right)^{j} F_{\gamma}^{(2)} D_{2}^{j} D_{1}^{p}
$$

$$
(\gamma=a \text { or } \gamma=\beta) \text {, }
$$

$$
R_{a}=\left(R_{a}^{(1)}\right)^{p}\left(R_{a}^{(2)}\right)^{q}, \quad F_{a}^{(i)}, F_{\beta}^{(i)} \in \mathscr{F}_{D_{i}}, R_{a}^{(i)}, R_{\beta}^{(i)} \in \mathscr{R}_{D_{i} .} \quad(i=1,2) .
$$

The Lagrange formula is of the form

$$
x D y-y D^{+} x=c_{D}^{-1}\left[D(x y)-f_{D}(x, y)\right] \text {. }
$$

The Green formula is of the form

$$
\begin{array}{r}
F_{\beta} R_{a}\left(x D y-y D^{+} x\right)=c_{D}^{-1}\left(F_{\beta}-F_{\alpha}\right)(x y)-F_{\beta} R_{\alpha} c_{D}^{-1} f(x, y) \\
\text { for } x, y \in \operatorname{dom} D .
\end{array}
$$

The proof follows from Corollary 2.8, Theorem 2.1 and Taylor formulae for operators $D_{1}$ and $D_{2}$ (cf. (1.2)). An immediate consequence is

Corollary 2.10. Let $X, D, c_{D}, \dot{f}_{D}, D^{+}, F_{a}, F_{\beta}, R_{\alpha}$ be defined as in Coroillary 2.9. Let

$$
Q(D)=\sum_{k=0}^{N} Q_{k} D^{k}, \quad Q^{+}(D)=\sum_{k=0}^{N}(-1)^{k} D^{k} Q_{k},
$$

where $Q_{k} \in L_{0}\left(\operatorname{dom} D^{N}\right)(k=0,1, \ldots, N)$ and $Q_{N}$ is invertible. Then the Green formula (2.3) holds. 
Corolidary 2.11. Let $X, D, c_{D}, f_{D}, D^{+}, F_{a}, F_{\beta}, R_{a}$ be defined as in Corrollary 2.9 (with $p=q=1$ ). Let

$$
\begin{aligned}
& P(D)=D_{2} D_{1}+A D_{1}+B D_{2}+C, \\
& P^{+}(D)=D_{2} D_{1}-D_{1} A-D_{2} B+C .
\end{aligned} \quad A, B, C \in L_{0}(X),
$$

Then the Green formula for all $x, y \in \operatorname{dom} D$ is of the form

$$
\begin{aligned}
& =c_{D_{1}}^{-1} c_{D_{2}}^{-1}\left(F_{\beta}-F_{\alpha}\right)(x y)+c_{D_{1}}^{-1}\left(F_{\beta}^{(1)}-F_{a}^{(1)}\right)(y A x)+c_{D_{2}}^{-1}\left(F_{\beta}^{(2)}-F_{a}^{(2)}\right)(y B x)+ \\
& +F_{\beta} R_{\alpha}\left\{c_{D_{1}}^{-1} c_{D_{2}}^{-1}\left[f_{D_{2}}(x, y)+D_{2} f_{D}(x, y)\right]+\left[\left(D_{1} x\right)\left(D_{2} y\right)+\left(D_{2} x\right)\left(D_{2} y\right)\right]+\right. \\
& \left.+c_{D_{1}}^{-1} f_{D_{1}}(A x, y)+c_{D_{2}}^{-1} f_{D_{2}}(B x, y)+[x, A] D_{1} y+[x, B] D_{2} y+y C x-x C y\right\},
\end{aligned}
$$

where $D=D_{2} D_{1}$, and

$$
\begin{gathered}
F_{\gamma}=F_{\gamma}^{(1)}+R_{\gamma}^{(1)} F_{\gamma}^{(2)} D_{1} \in \mathscr{F}_{D}=\mathscr{F}_{D_{2} D_{1}}(\gamma=\alpha \text { or } \gamma=\beta) \\
R_{a}=R_{\alpha}^{(1)} R_{a}^{(2)} \in \mathscr{R}_{D}=\mathscr{R}_{D_{1} D_{2}}, \quad F_{a}^{(i)}, F_{\beta}^{(i)} \in \mathscr{F}_{D_{i}}, R_{a}^{(i)} \in \mathscr{R}_{D_{i}} \quad(i=1,2) .
\end{gathered}
$$

Indeed, observe that for $x, y \in \operatorname{dom} D$ we have

$$
\begin{gathered}
x P(D) y-y P^{+}(D) x=x D_{2} D_{1} y-y D_{2} D_{1} x+ \\
\quad+x A D_{1} y-y D_{1} A x+x B D_{2} y-y D_{2} B x+x C y-y C x \\
=x D y-y D x+(A x) D_{1} y-y D_{1}(A x)+(B x)\left(D_{2} y\right)-y D_{2}(B x)+ \\
\quad+x C y-y C x+[x, A] D_{1} y+[x, B] D_{2} y \\
=c_{D}^{-1}\left[D(x, y)-f_{D}(x, y)\right]+c_{D_{1}}^{-1}\left[D_{1}(y A x)-f_{D_{1}}(A x, y)\right]+ \\
+c_{D_{2}}^{-1}\left[D_{2}(y B x)-f_{D_{2}}(B x, y)\right]+[x, A] D_{1} y+[x, B] D_{2} y+y C x-x C y .
\end{gathered}
$$

Further the proof is going in a similar way as in Corollaries 2.8 and 2.9.

Example 2.1. Suppose that $X=C(\Omega)$, where

$$
\begin{gathered}
\Omega=\{(t, s): 0 \leqslant t \leqslant a, 0 \leqslant s \leqslant b\}, \quad D=\frac{\partial^{2}}{\partial t \partial s}, \\
R_{0}=\int_{0}^{t} \int_{0}^{s}, \quad\left(F_{0} x\right)(t, s)=x(t, 0)+x(0, s)-x(0,0) .
\end{gathered}
$$

The operator $D=D_{2} D_{1}$, where $D_{2}=\partial / \partial t, D_{1}=\partial / \partial s$ is right invertible, $F_{0}$ is an initial operator for $D$ corresponding to its right inverse $R_{0}$. Moreover, $F_{0}$ is an initial operator induced by the classical Darboux problem for the operator $D$. To have Green formulae for the operator

$$
P(D)=\frac{\partial^{2}}{\partial t \partial s}+A \frac{\partial}{\partial t}+B \frac{\partial}{\partial s}+C, \quad A, B, C \in X,
$$


we have to find some more initial operators and right inverses for $D$ and to apply Corollary 2.10. Suppose then that we are given functions $g, h$ $\in C^{1}[0, a]$ such that $g^{\prime}(t)>0, h^{\prime}(t)>0$ of $0 \leqslant t \leqslant a, g(0)=h(0)=0$, $g(a)=h(a)=b$.

Consider the following operators defined for $x \in C^{1}(\Omega)$

$$
\begin{aligned}
& \left(F_{1} x\right)(t, s)=x\left(g^{-1}(s), s\right)+\int_{\sigma^{-1}(s)}^{t} x_{t}^{\prime}(p, g(p)) d p \\
& \left(F_{2} x\right)(t, s)=x\left(g^{-1}(s), s\right)-x\left(g^{-1}(s), 0\right)+x(t, 0), \\
& \left(F_{3} x\right)(t, s)=x(g(s), s)+\int_{g^{-1}(s)}^{t} x_{t}^{\prime}(p, h(p)) d p
\end{aligned}
$$

All these operators are initial operators for $D$ because they are projections onto $\operatorname{ker} D$. The operator $F_{1}$ is induced by the Cauchy problem for $D$, the operator $F_{2}$ is induced by the Picard problem for $D$, the operator $F_{3}$ is induced by the generalized Cauchy problem for $D$ (i.e. such a problem, where we are given values $x(t, g(t))$ and $x_{i}^{\prime}(t, h(t))$, cf. [7], [10]).

It is easy to verify that for all $x \in X$ we have

$$
\begin{aligned}
& \left(F_{1} R_{0} x\right)(t, s)=-\int_{0}^{\theta^{-1}(s)}\left[\int_{0}^{s} x(p, q) d q\right] d p-\int_{g^{-1}(s)}^{t}\left[\int_{0}^{g(p)} x(p, q) d q\right] d p \\
& \left(F_{2} R_{0} x\right)(t, s)=\int_{0}^{\sigma^{-1}(s)}\left[\int_{0}^{s} x(p, q) d q\right] d p \\
& \left(F_{3} R_{0} x\right)(t, s)=\int_{\sigma^{-1}(s)}^{0}\left[\int_{0}^{s} x(p, q) d q\right] d p-\int_{\theta^{-1}(s)}^{t}\left[\int_{0}^{h(p)} x(p, q) d q\right] d p
\end{aligned}
$$

Using these last expressions we can derive 3 different Green formulae for the operator $P(D)$ defined by formula (2.27).

Till now we have considered $D$-algebras over reals. Now we shall pass to $D$-algebras over the field $C$ of complexes. Write: $\boldsymbol{Y}=\boldsymbol{X} \oplus i \boldsymbol{X}$. The set $Y$ is an algebra with the addition, multiplication by scalar and multiplication of elements defined as follows:

$$
\begin{gathered}
(a+i b)+(c+i b)=(a+c)+i(b+d), \\
\lambda(a+i b)=\lambda a+i \lambda b \quad \text { for } a, b, c, d \in X, \lambda \in C, \\
(a+i b)(c+i d)=(a c-b d)+i(a d+b c) .
\end{gathered}
$$

Let $A=L(X)$ and let $u=x+i y$, where $x, y \in \operatorname{dom} A$. Write:

$$
A u=A x+i A y \text {. }
$$

By this definition $A \in L(Y)$. Indeed, $A(\lambda u)=\lambda A u$ for all $\lambda \in C, u \in Y$. Observe that $\overline{A u}=A \bar{u}$, where we write $\bar{u}=\overline{x+i y}=x-i y$. 
Indeed, we have $\bar{A} u=\overline{A x+i A y}=A x-i A y=A(x-i y)=A \bar{u}$.

It is easy to verify that $Y=X \oplus i X$ is a $D$-algebra over $C$, provided that $X$ is a $D$-algebra over $\boldsymbol{R}$. Initial operators and right inverses are extended according with formula (2.28). Write formula (1.7) in the complex case: For all $u, v \in \operatorname{dom} D$ (in $Y$ ) we have

$$
f_{D}(u, v)=D(u v)-c_{D}(u D v+v D u)
$$

where $c_{D}$ is a coefficient defined by formula (1.7) in the $D$-algebra $X$. By this definition, since $c_{D} \in R$, we have

$$
\overline{f_{D}(\bar{u}, \bar{v})}=f_{D}(u, v)=f_{D}(v, u) \quad \text { for all } u, v \in \operatorname{dom} D .
$$

Indeed,

$$
\begin{aligned}
\overline{f_{D}(\bar{u}, \bar{v})} & =\overline{D(\bar{u} \bar{v})}-\overline{c_{D}(\bar{u} D \bar{v}+\bar{v} D \bar{u})}=\overline{D(\bar{u} \bar{v})}-c_{D}(\overline{u D \bar{v}}+\overline{v D \bar{u}} \\
& =D(u v)-c_{D}(u D v+v D u)=f_{D}(u, v)=f_{D}(v, u) .
\end{aligned}
$$

Formula (2.29) implies that

$$
\bar{u} D v-\overline{v D^{+} u}=c_{D}^{-1} D(\bar{u} v)-f_{D}(\bar{u}, v) \quad \text { for all } u, v \in \operatorname{dom} D .
$$

Indeed, since $D^{+}=-D$, we find

$$
\bar{u} D v-\overline{v D^{+} u}=u D v-v D^{+} u=\bar{u} D v+v D \bar{u}=c_{D}^{-1}\left[D(\bar{u} v)-f_{D}(\bar{u}, v)\right] .
$$

We also have

$$
(\lambda D)^{+}=\bar{\lambda} D^{+} \quad \text { for all } \lambda \in C,
$$

i.e. the operator $D^{+}$is antilinear in the complex case.

Indeed, by formulae (2.30), (2.31) we have for $\hat{D}=\lambda D$

$$
\begin{aligned}
\bar{u}(\lambda D) v- & \overline{v(\lambda D)^{+} u}=\bar{u} \hat{D} v-v D^{+} u=c_{\hat{D}}^{-1}\left[\hat{D}(\bar{u} v)-f_{\hat{D}}(\bar{u}, v)\right] \\
& =c_{D}^{-1}\left[\lambda D_{\{}(u v)-f_{\lambda D}(\bar{u}, v)\right]=\lambda c_{D}^{-1}\left[D(\bar{u} v)-f_{D}(\bar{u}, v)\right] \\
& =\lambda\left(\bar{u} D v-v D^{+} u\right) .
\end{aligned}
$$

This implies that for all $\lambda \in C, u \in \operatorname{dom} D$ we have

$$
\bar{\lambda} D^{+} \bar{u}=\overline{\lambda D^{+} u}=\overline{(\lambda D)^{+}} u=(\lambda D)^{+} u \text {. }
$$

The arbitrariness of $u \in \operatorname{dom} D$ implies (2.31).

In particular, we have

$$
(i D)^{+}=i D \text {. }
$$

All further considerations are going in similar as in the real case. In particular, the Green formula (2.3) in the complex case is of the form

$$
=\sum_{j=0}^{N-1}(-1)^{j} e_{D}^{-(j+1)}\left(F_{\beta}-F_{a}\right)\left(y Q_{j+1} \bar{x}\right)+F_{\beta} R_{\alpha}\left[g_{Q(D)}(\bar{x}, y)+h_{Q(D)}(\bar{x}, y)\right],
$$

where $g_{Q(D)}, h_{Q(D)}$ are defined by formulae $(2.4),(2.5)$. 
Indeed, if we put $x$ instead of $\bar{x}$ and we shall make use of (2.29), we obtain that

$$
\overline{Q^{+}(D) x}=\overline{\sum_{k=0}^{N}(-1)^{k} D^{k} Q_{k} x}=\sum_{k=0}^{N}(-1)^{k} D^{k} Q_{k} \bar{x}
$$

which implies formula (2.33).

\section{References}

[1] Z. Dudek, Some properties of Wronskian in D-R spaces of the type Q-L, Part I, Demonstratio Math. 11 (1978), p. 1115-1130, Part II, ibidem 13 (1980), p. 987993.

[2] -, Decompositions of quasi-Leibniz $D-R$ algebras (submitted to printing).

[3] W. Haack, W. Wendland, Partial and Pfaffian differential equations, Pergamon Press, Oxford-New York, 1972.

[4] P. Hartman, Ordinary differential equations, J. Wiley and Sons, New YorkLondon-Sydney, 1964.

[5] E. Kamke, Differentialgleichungen. Lösungsmethoden und Lösungen. Akademische Verlagsgesellschaft. Gesst and Portig K.-G. Leipzig, 1962.

[6] S. Kurcyusz, Necessary conditions of optimality for problems with constraints in a function space (in Polish), Ph. dissertation, Institute of Automatics, Technical University of Warsaw, Warszawa, 1972.

[7] D. Przeworska-Rolewicz, Algebraic theory of right invertible operators, Studia Math. 48 (1979), p. 129-144.

[8] -, Admissible initial operators for superpositions of right invertible operators, Ann. Polon. Math. 33 (1976), p. 113-120.

[9] -, On trigonometric identity for right invertible operators, Comm. Math. 21 (1978), p. 267-277.

[10] -, Introduction to algebraic analysis and its applications (in Polish), WNT. Publishers in Sciences and Technology, Warszawa, 1979.

[11] -, Shifts and periodicity for right invertible operators, Research Notes in Mathematics, '43, Pitman Publish. Ltd., Boston-London-Melbourne, 1980.

[12] -, Right inverses and Volterra operators, Journ. of Integral Equations 2 (1980), p. 45-46.

[13] -, Picone's identity for right invertible operators, Proceedings of the Conference "Selected Topics in Applied Analysis", GMD, Bonn, October 1979.

[14] -, Operational calculus and algebraic analysis. Generalized functions and operational calculus, Proceedings of the Conference on Generalized Functions and Operational Calculi, Varna, September 29-October, 1975. Publish. House of the Bulg. Acad. of Sciences, Sofia, 1979.

[15] -, Integration of a unit in linear rings with right invertible operators, C. R. Math. Rep. Acad. Sci. Canada 1 (1979), p. 227-230.

[16] -, Concerning Euler-Lagrange equation in algebras with right invertible operators, Proceedings of "Game Theory and Related Topics", Hagen-Bonn, Oetober 6-9, 1980, North-Holland Publ. Comp., Amsterdam, 1981.

[17] -, Green formula and duality for right invertible operators, Preprint $\mathrm{nr} 215$, Institute of Mathematics, Polish Academy of Sciences, May 1980. 
[18] D. Przeworska-Rolewicz and H. von Trotha, Right inverses in $D-R$ algebras with unit, Journ. of Integral Equations (1981), p. 245-259.

[19] S. Schwabik, M. Tvrdý, O. Vejvoda, Differential and integral equations. Boundary value problems and adjoints, Academia, Praha, 1979.

INSTITUTE OF MATHEMATICS

POLISH ACADEMY OF SCIENCES

SNIADECKICH 8, 00-950 WARSZAWA

POLAND

Reģu par la Rédaction le 19.09.1980 